\title{
GALACTOSE DISAPPEARANCE FROM THE BLOOD STREAM. CALCULATION OF A GALACTOSE REMOVAL CONSTANT AND ITS APPLICATION AS A TEST FOR LIVER FUNCTION
}

\author{
By HENRY COLCHER, ARTHUR J. PATEK, JR., AND FORREST E. KENDALL \\ (From the Research Scrvice, First (Columbia) Division, Goldwater Memorial Hospital, Depart- \\ ment of Hospitals, and the College of Physicians and Surgeons, Columbia University, New \\ York City)
}

(Received for publication April 6, 1946)

The use of galactose for testing the glycogenic function of the liver was suggested by Bauer in 1906 (1). Galactose is suitable for this purpose since its utilization depends upon the functional integrity of the liver, since it is non-toxic, and since its concentration can be determined readily in blood and urine ( 2 to 7 ).

Bauer's test measured the urinary excretion of galactose during the 5-hour period following ingestion of the sugar. An excessive excretion of galactose was interpreted to indicate liver damage. However, there are serious disadvantages to this procedure: the method lacks sensitivity, and it is subject to errors introduced by changes in gastrointestinal and renal functions.

To overcome the disadvantages of an oral test Jankelson, Segal and Aisner (8) introduced an intravenous galactose tolerance test. Their technique was modified by King and Aitkin (9) and by Bassett, Althausen and Coltrin (10). These studies defined a range of normal and abnormal values for blood galactose at a given time after intravenous injection of the substance. For example, Bassett, Althausen, and Coltrin found that in normal adults no galactose remained in the blood stream 75 minutes after the injection of 0.5 gram galactose per $\mathrm{kgm}$. body weight. Appreciable concentrations of galactose were still present at this time in patients with liver damage. Because of these findings, the value at 60 or 75 minutes was proposed as a diagnostic index for the presence or absence of liver damage. However, interpretation of the significance of a single value presents certain drawbacks. The blood galactose concentration at a given moment depends not only upon the function of the liver, but also upon blood and tissue space volumes. The value for galactose concentration at 60 or 75 minutes after intravenous injec- tion represents the result of removal of galactose from the blood stream, but does not measure the velocity of the process.

In the present study, repeated determinations of blood galactose were made during a period of $1 \frac{1}{2}$ hours after intravenous injection of the substance. A constant expressing the rate of disappearance of galactose from the blood stream was derived from the data. Comparison was made of the values of this constant in normal subjects, in patients without evidence of liver damage, and in patients with various liver diseases. The value of this constant was also compared with the results of other liver function tests.

\section{METHODS}

A 50 per cent galactose solution ${ }^{1}$ was prepared according to the method of King and Aitken (9). After testing the solution for sterility, it was stored at $37^{\circ} \mathrm{C}$. in an incubator to prevent crystallization. The galactose test was performed usually about 2 hours after the patient had received breakfast. First, blood was drawn to furnish a blank for the non-galactose, non-fermenting copper reducing substances. Directly thereafter galactose solution corresponding to 0.5 gram per $\mathrm{kgm}$. was injected intravenously in 2 to 3 minutes. Venous blood samples for galactose determination were drawn from the opposite arm at 15-minute intervals over a period of $1 \frac{1 / 2}{2}$ hours. Urine was collected as voided over a 4-hour period. The determination of blood galactose was based upon Benedict's method for blood sugar (11), after removal of glucose by fermentation according to Raymond and Blanco's method (12). The procedure was as follows: To $2 \mathrm{ml}$. of whole oxalated blood were added $12 \mathrm{ml}$. of 20 per cent suspension of fresh baker's yeast (washed 7 times). Fermentation was complete in 10 minutes. To the mixture were then added $6 \mathrm{ml}$. of a protein-precipitating mixture containing equal amounts of 10 per cent sodium tungstate and $2 / 3 \mathrm{~N}$ sulfuric acid. The remainder

1 Two brands were used, galactose, C. P. Amend Drug and Chemical Co., New York City, and galactose, Merck and Co., Rahway, N. J. 
of the procedure, i.e., heating, cooling, colorimetry, follows Benedict's method. However, galactose standards ( 10 or $20 \mathrm{mgm}$. per cent concentrations) were employed instead of glucose standards. The determination of galactose in the urine was done by Benedict's quantitative method for urinary glucose. A conversion factor of 1.14 was used.

Derivation of an expression for the rate of galactose removal from the blood

Figure 1 shows values obtained on a representative case of passive congestion of the liver. When the successive values for blood galactose are plotted against time, a smooth curve is obtained. The shape of the curve indicates that the rate of removal of galactose is not constant, but decreases as the concentration of the galactose in the blood

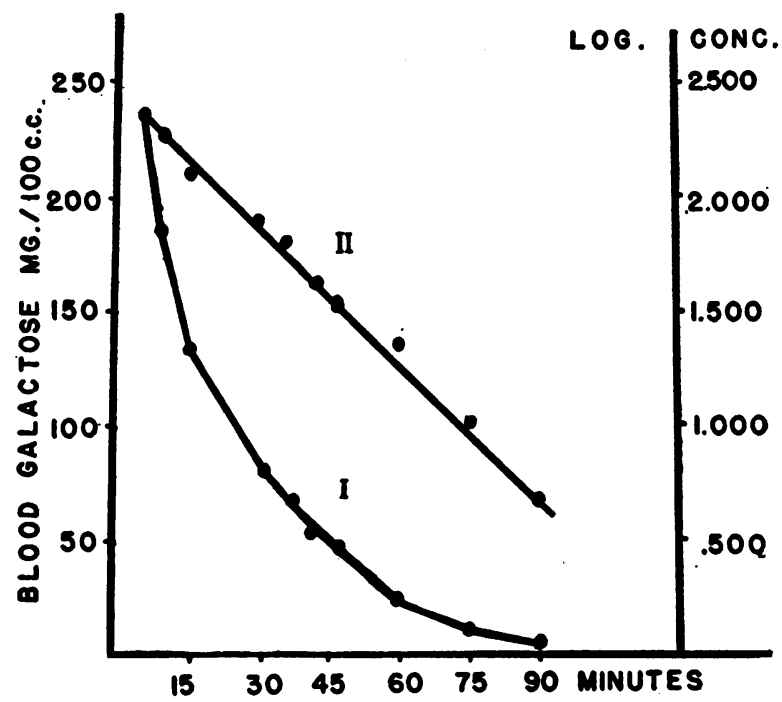

Fig. 1. Curves Showing the Disappearance of Galactose from the BLOOd AFTER I.v. InJection OF 0.5 Gram of Galactose per Kgm. in Patient M. W.

Diagnosis, chronic passive congestion of the liver due to heart failure. In curve I the blood galactose concentrations, in mgm. per $100 \mathrm{ml}$., are plotted against time in minutes. In curve II the logarithms of these concentrations are plotted against time.

decreases. However, when the logarithm of blood concentration is plotted against time, a straight line is obtained. This straight line may be interpreted to indicate that the rate of removal of galactose from the blood is directly proportional to the concentration in the blood. This can be expressed mathematically as:

$$
-\frac{d C}{d t}=K C
$$

In this equation " $C$ " represents the concentration of galactose in the blood at time " $t$ " and " $K$ " represents the proportionality constant. The value of $K$ may be obtained from the slope of the line in Figure 1, or may be calculated from the equation obtained by integration of the above expression.

Integration leads to this equation :

$$
K=\frac{2.3\left(\log C_{1}-\log C_{2}\right)}{t_{2}-t_{1}}
$$

$C_{1}$ and $C_{2}$ are the concentrations at the times, $t_{1}$ and $t_{2}$. If $t$ is expresed in minutes, $K$ represents the fraction of the total amount of galactose present at any given time that is removed from the blood stream each minute. For convenience, the decimal fraction obtained from this equation is multiplied by 100 so that the numerical value expresses the percentage of the amount present that is removed each minute. This might be termed "The Galactose Removal Constant" or the G.R.C.

Although in this study 6 or 7 blood specimens were obtained from each patient at 15-minute intervals in order to establish the validity of this constant, it was found that only 2 values are required for its calculation. The galactose removal constant may be obtained with sufficient accuracy for clinical purposes from blood concentrations at 15 and 45 minutes after completion of the intravenous injection. Under these conditions the equation may be simplified to read as follows: G.R.C. $=7.6$ (log concentration at 15 minutes $\log$ concentration at 45 minutes).

\section{Galactose Removal Constant in various conditions}

Tests were performed on 64 patients. ${ }^{2}$ The case material included 6 groups: (1) Control group of normal adults (24 to 40 years) and patients with no clinical evidence of liver disease. (2) Patients with decompensated cirrhosis of the liver, i.e., patients with ascites or jaundice. (3) Patients with cirrhosis of the liver without signs of decompensation. (4) Patients with chronic passive congestion of the liver. (5) Patients with acute hepatitis. (6) Miscellaneous group of patients with liver diseases due to other causes. The grouping of patients was made mainly on the basis of clinical findings. Admittedly, one cannot be entirely certain of the diagnosis. For example, patient M. C.

\footnotetext{
2 The authors wish to thank Dr. Franklin M. Hanger who made available 5 patients for this study.
} 
TABLE I

Normal adults

\begin{tabular}{|c|c|c|c|c|c|c|c|c|c|c|c|c|c|c|c|c|}
\hline \multirow{3}{*}{ Name } & \multirow{3}{*}{$\begin{array}{l}\text { Galact. } \\
\text { amount } \\
\text { inject. }\end{array}$} & \multicolumn{6}{|c|}{ Blood galactose at: } & \multirow{3}{*}{$\begin{array}{c}\text { Total } \\
\text { excret. } \\
\text { In } \\
\text { urine }\end{array}$} & \multirow{3}{*}{$\begin{array}{l}\text { Galact. } \\
\text { removal } \\
\text { const. }\end{array}$} & \multirow{3}{*}{$\begin{array}{c}\text { Proth. } \\
\text { time }\end{array}$} & \multirow{3}{*}{$\begin{array}{l}\text { B.S.P. } \\
30 \text { min. }\end{array}$} & \multirow{3}{*}{$\begin{array}{l}\text { Ceph. } \\
\text { floc. }\end{array}$} & \multirow{3}{*}{$\begin{array}{l}\text { Serum } \\
\text { bilirub }\end{array}$} & & & \multirow{3}{*}{$\begin{array}{l}\text { Urine } \\
\text { urobi- } \\
\text { linogen }\end{array}$} \\
\hline & & 15 & & & & & & & & & & & & & & \\
\hline & & \multicolumn{6}{|c|}{ Minutes after I. V. injection } & & & & & & & Alb. & Glob. & \\
\hline & grams & \multicolumn{6}{|c|}{ mgm. per $100 \mathrm{ml}$. } & grams & $\begin{array}{c}\text { per cent } \\
\text { per } \\
\text { min. }\end{array}$ & seconds & $\begin{array}{l}\text { per } \\
\text { cent } \\
\text { ret. }\end{array}$ & & $\begin{array}{c}\text { mgm. } \\
\text { per } \\
100 \mathrm{ml} .\end{array}$ & \multicolumn{2}{|c|}{$\begin{array}{l}\text { grams per } \\
100 \text { ml. }\end{array}$} & $\begin{array}{c}\text { perm. } \\
\text { hour }\end{array}$ \\
\hline $\begin{array}{l}\text { H. M. } \\
\text { H. C. } \\
\text { R. L. } \\
\text { A. L. } \\
\text { A. P. } \\
\text { K. S. }\end{array}$ & $\begin{array}{l}45 \\
42 \\
27.5 \\
34 \\
32 \\
40\end{array}$ & $\begin{array}{l}156 \\
139 \\
107 \\
177 \\
105 \\
186\end{array}$ & $\begin{array}{r}87 \\
67 \\
53 \\
114 \\
48 \\
71\end{array}$ & $\begin{array}{l}34 \\
17 \\
16 \\
50 \\
13 \\
11\end{array}$ & $\begin{array}{r}18 \\
0 \\
4 \\
17 \\
4 \\
4\end{array}$ & $\begin{array}{l}2 \\
0 \\
0 \\
4 \\
0 \\
0\end{array}$ & $\begin{array}{l}\mathbf{0} \\
\mathbf{0} \\
\mathbf{0} \\
\mathbf{0} \\
\mathbf{0} \\
\mathbf{0}\end{array}$ & $\begin{array}{l}6.00 \\
3.34 \\
4.10 \\
3.48 \\
4.27\end{array}$ & $\begin{array}{l}5.1 \\
7.1 \\
6.3 \\
4.2 \\
6.9 \\
9.5\end{array}$ & $\begin{array}{l}18 \\
14 \\
17 \\
16 \\
17 \\
17\end{array}$ & $\begin{array}{l}7.5 \\
8 \\
4 \\
8 \\
2.5 \\
6\end{array}$ & $\begin{array}{l}0 \\
0 \\
0 \\
0 \\
0 \\
2+\end{array}$ & $\begin{array}{l}1.00 \\
1.20 \\
1.65 \\
1.45 \\
1.00 \\
0.80\end{array}$ & $\begin{array}{l}5.4 \\
5.3 \\
4.9 \\
5.2 \\
5.3 \\
5.2\end{array}$ & $\begin{array}{l}1.6 \\
2.5 \\
2.4 \\
2.2 \\
2.1 \\
2.0\end{array}$ & $\begin{array}{r}.39 \\
0.50 \\
0.41\end{array}$ \\
\hline
\end{tabular}

Patients with no clinical evidence of liver disease

\begin{tabular}{|c|c|c|c|c|c|c|c|c|c|c|c|c|c|c|c|}
\hline $\begin{array}{l}\text { M. W. } \\
\text { J.S.S. } \\
\text { E.F. } \\
\text { M.C. }\end{array}$ & $\begin{array}{l}38.5 \\
32 \\
25 \\
22\end{array}$ & $\begin{array}{l}187 \\
146 \\
160 \\
76^{*}\end{array}$ & $\begin{array}{l}95 \\
67 \\
70 \\
39\end{array}$ & $\begin{array}{l}32 \\
15 \\
31 \\
20\end{array}$ & $\begin{array}{r}4 \\
0 \\
11 \\
0\end{array}$ & $\begin{array}{l}0 \\
0 \\
0 \\
0\end{array}$ & $\begin{array}{l}0 \\
0 \\
0 \\
0\end{array}$ & $\begin{array}{l}3.18 \\
3.76 \\
3.07 \\
1.87\end{array}$ & $\begin{array}{l}5.9 \\
7.6 \\
5.5 \\
4.8\end{array}$ & $\begin{array}{l}16 \\
16 \\
12 \\
18\end{array}$ & $\begin{array}{r}5 \\
10 \\
5 \\
4\end{array}$ & $\begin{array}{l}\mathbf{0} \\
2+ \\
0 \\
3+\end{array}$ & $\begin{array}{l}1.35 \\
\text { I.I. } 3 \\
1.15\end{array}$ & $\begin{array}{l}5.1 \\
4.8 \\
5.3 \\
3.9\end{array}$ & $\begin{array}{l}2.7 \\
2.4 \\
2.3 \\
4.0\end{array}$ \\
\hline
\end{tabular}

Decompensated cirrhosis of the liver

\begin{tabular}{|c|c|c|c|c|c|c|c|c|c|c|c|c|c|c|c|c|}
\hline $\begin{array}{l}\text { S. P. } \\
\text { M. D. } \\
\text { T.S. } \\
\text { S. B. } \\
\text { S. K. } \\
\text { C. G. } \\
\text { E. F. } \\
\text { K. K. } \\
\text { N. K. } \\
\text { W. H. } \\
\text { R. P. } \\
\text { V. F. } \\
\text { C. P. } \\
\text { K. K. } \\
\mathbf{P} . \mathbf{B} . \\
\text { J.A. } \\
\text { M. D. } \\
\text { E. P. } \\
\text { A. G. }\end{array}$ & $\begin{array}{l}26 \\
27 \\
34.5 \\
29 \\
31.5 \\
40 \\
33 \\
27 \\
36 \\
33.5 \\
26 \\
28 \\
34 \\
30 \\
36 \\
38 \\
23 \\
27 \\
33\end{array}$ & $\begin{array}{r}189 \\
190 \\
157 \\
131 \\
109 \\
175 \\
147 \\
97 \\
192 \\
109 \\
141 \\
162 \\
123 \\
139 \\
173 \\
149 \\
113 \\
143 \\
182\end{array}$ & $\begin{array}{r}134 \\
130 \\
106 \\
106 \\
89 \\
119 \\
107 \\
66 \\
154 \\
72 \\
90 \\
106 \\
87 \\
110 \\
145 \\
90 \\
91 \\
108 \\
141\end{array}$ & $\begin{array}{r}83 \\
110 \\
96 \\
76 \\
65 \\
92 \\
67 \\
39 \\
106 \\
53 \\
56 \\
75 \\
65 \\
89 \\
117 \\
69 \\
69 \\
81 \\
118\end{array}$ & $\begin{array}{l}53 \\
79 \\
67 \\
63 \\
42 \\
73 \\
51 \\
18 \\
68 \\
31 \\
54 \\
35 \\
59 \\
78 \\
91 \\
40 \\
42 \\
66 \\
97\end{array}$ & $\begin{array}{l}16 \\
61 \\
44 \\
38 \\
27 \\
46 \\
32 \\
9 \\
30 \\
14 \\
25 \\
14 \\
46 \\
70 \\
77 \\
21 \\
28 \\
44 \\
81\end{array}$ & $\begin{array}{r}9 \\
39 \\
13 \\
34 \\
16 \\
24 \\
20 \\
8 \\
16 \\
5 \\
13 \\
2 \\
30 \\
61 \\
56 \\
15 \\
3 \\
22 \\
63\end{array}$ & $\begin{array}{l}1.60 \\
4.30 \\
2.04 \\
4.80 \\
4.65 \\
7.20 \\
1.70 \\
4.10 \\
4.80 \\
2.28 \\
4.10 \\
2.66 \\
\\
3.97 \\
3.40 \\
3.11 \\
5.43 \\
3.60\end{array}$ & $\begin{array}{l}2.73 \\
1.82 \\
1.65 \\
1.80 \\
1.70 \\
2.10 \\
2.60 \\
\mathbf{3 . 0 0} \\
2.00 \\
2.60 \\
3.06 \\
2.56 \\
2.12 \\
1.46 \\
1.30 \\
2.56 \\
1.65 \\
1.9 \\
1.5\end{array}$ & $\begin{array}{l}21 \\
21 \\
16 \\
23 \\
22 \\
32 \\
20 \\
19 \\
16 \\
19 \\
24 \\
20 \\
18 \\
30 \\
18 \\
24 \\
20 \\
27 \\
17\end{array}$ & $\begin{array}{c}52.5 \\
46 \\
28 \\
41 \\
20 \\
45 \\
33 \\
45 \\
20 \\
27 \\
63 \\
56 \\
100 \\
67 \\
100 \\
55 \\
93 \\
87\end{array}$ & $\begin{array}{l}+ \\
3+ \\
3+ \\
3+ \\
3+ \\
3+ \\
3+ \\
3+ \\
4+ \\
3+ \\
4+ \\
4+ \\
4+ \\
4+ \\
4+ \\
4+ \\
3+ \\
4+\end{array}$ & $\begin{array}{l}2.0 \\
1.1 .6 \\
1.5 \\
1.1 .8 \\
0.90 \\
0.70 \\
0.20 \\
2.25 \\
0.20 \\
1.00 \\
1.10 \\
1.85 \\
1.55 \\
8.10 \\
1.25 \\
3.20 \\
0.95 \\
3.00 \\
1.45\end{array}$ & $\begin{array}{l}3.3 \\
3.6 \\
3.8 \\
4.2 \\
3.7 \\
3.3 \\
3.2 \\
3.8 \\
4.9 \\
2.5 \\
2.7 \\
4.2 \\
2.5 \\
2.2 \\
3.9 \\
3.2 \\
2.8 \\
2.3 \\
2.7\end{array}$ & $\begin{array}{l}3.5 \\
3.5 \\
2.5 \\
2.8 \\
2.2 \\
3.3 \\
1.8 \\
2.6 \\
3.2 \\
3.6 \\
4.3 \\
3.8 \\
3.3 \\
3.5 \\
3.0 \\
3.8 \\
4.3 \\
5.9 \\
3.3\end{array}$ & $\begin{array}{l}0.20 \\
0.32 \\
0.75 \\
0.96 \\
0.37 \\
1.34 \\
0.90 \\
0.36 \\
0.50 \\
0.76 \\
0.52 \\
0.68 \\
1.02 \\
0.60 \\
1.19 \\
0.93 \\
0.42 \\
0.41\end{array}$ \\
\hline
\end{tabular}

Cirrhosis compensated

\begin{tabular}{|c|c|c|c|c|c|c|c|c|c|c|c|c|c|c|c|c|}
\hline $\begin{array}{l}\text { C. M. } \\
\text { T. A. } \\
\text { H. S. } \\
\text { J. G. } \\
\text { C. B. } \\
\text { M. O. } \\
\text { A. H. }\end{array}$ & $\begin{array}{l}\mathbf{4 0} \\
35 \\
28 \\
41 \\
37 \\
32 \\
33.5\end{array}$ & $\begin{array}{l}171 \\
180 \\
193 \\
190 \\
200 \\
200 \\
169\end{array}$ & $\begin{array}{l}133 \\
118 \\
113 \\
122 \\
146 \\
160 \\
147\end{array}$ & $\begin{array}{r}90 \\
51 \\
52 \\
75 \\
92 \\
132 \\
86\end{array}$ & $\begin{array}{l}57 \\
20 \\
19 \\
20 \\
53 \\
96 \\
57\end{array}$ & $\begin{array}{r}29 \\
6 \\
11 \\
7 \\
38 \\
57 \\
37\end{array}$ & $\begin{array}{r}21 \\
3 \\
3 \\
6 \\
22 \\
36 \\
16\end{array}$ & $\begin{array}{l}5.29 \\
3.69 \\
4.03 \\
3.23 \\
7.65 \\
4.39 \\
\mathbf{3 . 3 6}\end{array}$ & $\begin{array}{l}2.14 \\
4.16 \\
3.96 \\
3.10 \\
2.60 \\
1.38 \\
2.23\end{array}$ & $\begin{array}{l}19 \\
18 \\
17 \\
19 \\
17 \\
24 \\
20\end{array}$ & $\begin{array}{r}80 \\
30 \\
49 \\
44 \\
51 \\
100 \\
67\end{array}$ & $\begin{array}{l}0+ \\
4+ \\
2+ \\
3+ \\
3+\end{array}$ & $\begin{array}{l}1.5 \\
1.05 \\
1.45 \\
1.35 \\
0.50 \\
3.55 \\
1.85\end{array}$ & $\begin{array}{l}4.3 \\
4.6 \\
5.1 \\
4.7 \\
4.5 \\
3.9 \\
5.3\end{array}$ & $\begin{array}{l}3.0 \\
2.4 \\
2.7 \\
2.8 \\
3.4 \\
5.1 \\
3.2\end{array}$ & 0.40 \\
\hline
\end{tabular}

Chronic passive congestion of the liver

\begin{tabular}{|c|c|c|c|c|c|c|c|c|c|c|c|c|c|c|c|}
\hline $\begin{array}{l}\text { W. B. } \\
\text { V. B. } \\
\text { w. T. } \\
\text { M.C. } \\
\text { J. H. } \\
\text { D.S. } \\
\text { C. O. } \\
\text { L. W. } \\
\text { C. E. } \\
\text { M. W. }\end{array}$ & $\begin{array}{l}37 \\
35 \\
32 \\
39 \\
37 \\
36 \\
45 \\
29 \\
29 \\
28\end{array}$ & $\begin{array}{l}172 \\
193 \\
166 \\
141 \\
162 \\
148 \\
193 \\
165 \\
154 \\
134\end{array}$ & $\begin{array}{l}102 \\
134 \\
118 \\
101 \\
104 \\
118 \\
156 \\
100 \\
100 \\
81 \mathrm{tt}\end{array}$ & $\begin{array}{c}61 \\
89 \\
80 \\
57 \\
61 \\
84 \\
114 \\
41 * * \\
57 \\
48+t+\end{array}$ & $\begin{array}{l}30 \\
46^{*} \\
47 \\
37 \\
18 \\
64 \\
90 \\
15 \\
23\end{array}$ & $\begin{array}{c}19 \\
31 \\
20 \\
6 \\
6 \\
41 \\
64 \\
16 \dagger \\
0 \\
11\end{array}$ & $\begin{array}{r}2 \\
15 \\
4 \\
0 \\
3 \\
14 \\
50 \\
0 \\
0 \\
5\end{array}$ & $\begin{array}{l}4.06 \\
0.67 \\
3.55 \\
4.36 \\
4.70 \\
3.93 \\
3.65 \\
4.79 \\
4.67 \\
1.30\end{array}$ & $\begin{array}{l}3.42 \\
2.56 \\
2.42 \\
3.02 \\
3.2 \\
1.96 \\
1.76 \\
3.97 \\
2.4 \\
4\end{array}$ & $\begin{array}{l}21 \\
13 \\
16 \\
14 \\
17 \\
11 \\
17 \\
20 \\
15\end{array}$ & $\begin{array}{l}16 \\
42.5 \\
35.5 \\
49 \\
19 \\
51 \\
33 \\
33 \\
10\end{array}$ & $\begin{array}{c}2+ \\
+ \\
+ \\
+ \\
4\end{array}$ & $\begin{array}{l}0.70 \\
0.40 \\
0.70 \\
0.50 \\
0.15 \\
0.75 \\
1.55 \\
1.75\end{array}$ & $\begin{array}{l}4.3 \\
3.9 \\
4.1 \\
3.6 \\
4.0 \\
4.1 \\
4.8 \\
4.7 \\
5.0 \\
4.2\end{array}$ & $\begin{array}{l}2.5 \\
3.7 \\
2.8 \\
2.9 \\
2.4 \\
3.3 \\
2.7 \\
3.4 \\
3.1 \\
4.4\end{array}$ \\
\hline
\end{tabular}

* 62 minutes. ** 50 minutes. $\quad 70$ minutes. †† 32 minutes. †† 47 minutes. 
TABLE I (continued)

Acute hepatitis

\begin{tabular}{|c|c|c|c|c|c|c|c|c|c|c|c|c|c|c|c|c|c|}
\hline \multirow{3}{*}{ Name } & \multirow{3}{*}{$\begin{array}{l}\text { Galact. } \\
\text { amount } \\
\text { inject. }\end{array}$} & \multicolumn{6}{|c|}{ Blood galactose at: } & \multirow{3}{*}{$\begin{array}{c}\text { Total } \\
\text { excret. } \\
\text { in } \\
\text { urine }\end{array}$} & \multirow{3}{*}{$\begin{array}{l}\text { Galact. } \\
\text { removal } \\
\text { const. }\end{array}$} & \multirow{3}{*}{$\begin{array}{c}\text { Proth. } \\
\text { time }\end{array}$} & \multirow{3}{*}{$\begin{array}{l}\text { B.S.P. } \\
30 \\
\text { min. }\end{array}$} & \multirow{3}{*}{$\begin{array}{l}\text { Ceph. } \\
\text { floc. }\end{array}$} & \multirow{3}{*}{$\begin{array}{l}\text { Serum } \\
\text { bilirub }\end{array}$} & \multirow{2}{*}{\multicolumn{2}{|c|}{ Serum }} & \multirow{3}{*}{$\begin{array}{c}\text { Urine } \\
\text { urobi- } \\
\text { linogen }\end{array}$} & \multirow{3}{*}{ Remarks } \\
\hline & & 15 & 30 & 45 & 60 & 75 & 90 & & & & & & & & & & \\
\hline & & \multicolumn{6}{|c|}{ Minutes after I. V. injection } & & & & & & & Alb. & Glob. & & \\
\hline $\begin{array}{l}\text { E.S. } \\
\text { E.S. } \\
\text { E.S. } \\
\text { T. B. } \\
\text { M.T. } \\
\text { M. I. } \\
\text { G. C. } \\
\text { J.O. } \\
\text { L. } \mathbf{w} .\end{array}$ & $\begin{array}{c}\text { grams } \\
27 \\
27 \\
26 \\
34 \\
27 \\
22 \\
25 \\
31 \\
32\end{array}$ & $\begin{array}{l}184 \\
188 \\
131 \\
165 \\
186 \\
139 \\
134 \\
146 \\
161\end{array}$ & $\begin{array}{r}139 \\
145 \\
69 \\
124 \\
142 \\
112 \\
99 \\
115\end{array}$ & $\begin{array}{r}104 \\
104 \\
20 \\
107 \\
121 \\
92 \\
67 \\
63 \\
80\end{array}$ & $\begin{array}{l}69 \\
57 \\
0 \\
92 \\
80 \\
86 \\
21 \\
46\end{array}$ & $\begin{array}{r}53 \\
36 \\
0 \\
74 \\
63 \\
77 \\
10 \\
20\end{array}$ & $\begin{array}{r}28 \\
\\
62 \\
37 \\
68 \\
1 \\
9 \\
2\end{array}$ & $\begin{array}{c}\text { grams } \\
5.74 \\
3.61 \\
1.33 \\
1.88 \\
2.97 \\
5.97 \\
4.76\end{array}$ & $\begin{array}{c}\text { per cent } \\
\text { per } \\
\text { min. } \\
1.9 \\
1.94 \\
6.2 \\
1.44 \\
1.45 \\
1.37 \\
2.36 \\
2.8 \\
2.36\end{array}$ & $\begin{array}{c}\text { sec- } \\
\text { onds } \\
23 \\
16 \\
35 \\
18 \\
23\end{array}$ & $\begin{array}{l}\text { per } \\
\text { cent } \\
\text { ret. } \\
42 \\
0 \\
100 \\
51 \\
81 \\
68\end{array}$ & $\begin{array}{l}4+ \\
4+ \\
0 \\
4+ \\
4+ \\
4+ \\
4+ \\
4+ \\
+\end{array}$ & $\begin{array}{c}\underset{\text { mgm. }}{\text { per }} \\
100 \mathrm{ml} . \\
20 \\
3.1 \\
15 \\
11 \\
9.80 \\
2.25\end{array}$ & $\begin{array}{r}\begin{array}{c}8 r a m \\
100\end{array} \\
4.1 \\
\\
4.2 \\
3.5 \\
4.3 \\
4.8\end{array}$ & $\begin{array}{l}3.6 \\
3.7 \\
3.2 \\
2.6\end{array}$ & $\begin{array}{c}\text { mgm. } \\
\text { per } \\
\text { hour } \\
+1: 64 \\
\text { Neg. }\end{array}$ & $\begin{array}{l}2 \text { nd day of jaundice } \\
10 \text { days later } \\
3 \text { mos. after recovery }\end{array}$ \\
\hline
\end{tabular}

Miscellaneous

\begin{tabular}{|c|c|c|c|c|c|c|c|c|c|c|c|c|c|c|c|c|c|}
\hline \multirow{3}{*}{ Name } & \multirow{3}{*}{$\begin{array}{l}\text { Galact. } \\
\text { amount } \\
\text { inject. }\end{array}$} & \multicolumn{6}{|c|}{ Blood galactose at: } & \multirow{3}{*}{$\begin{array}{l}\text { Total } \\
\text { excret. } \\
\text { in } \\
\text { urine }\end{array}$} & \multirow{3}{*}{$\begin{array}{l}\text { Galact. } \\
\text { removal } \\
\text { const. }\end{array}$} & \multirow{3}{*}{$\begin{array}{c}\text { Proth. } \\
\text { time }\end{array}$} & \multirow{3}{*}{$\begin{array}{l}\text { B.S.P. } \\
30 \\
\text { min. }\end{array}$} & \multirow{3}{*}{$\begin{array}{l}\text { Ceph. } \\
\text { floc. }\end{array}$} & \multirow{3}{*}{$\begin{array}{l}\text { Serum } \\
\text { bilirub }\end{array}$} & & & \multirow{3}{*}{$\begin{array}{l}\text { Urine } \\
\text { urobi- } \\
\text { linogen }\end{array}$} & \multirow{3}{*}{ Diagnosis } \\
\hline & & 15 & 30 & 15 & 60 & 75 & $\infty$ & & & & & & & set & um & & \\
\hline & & \multicolumn{6}{|c|}{ Minutes after I. V. injection } & & & & & & & Alb. & Glob. & & \\
\hline & grams & \multicolumn{6}{|c|}{ mgm. per $100 \mathrm{ml}$. } & grams & $\begin{array}{c}\text { per cent } \\
\text { per } \\
\text { min. }\end{array}$ & $\begin{array}{l}\text { sec- } \\
\text { onds }\end{array}$ & $\begin{array}{l}\text { per } \\
\text { cent } \\
\text { ret. }\end{array}$ & & $\begin{array}{c}\text { mgm. } \\
\text { per } \\
100 \mathrm{ml} .\end{array}$ & \multicolumn{2}{|c|}{$\begin{array}{l}\text { grams per } \\
100 \text { ml. }\end{array}$} & $\begin{array}{c}\text { mem. } \\
\text { per } \\
\text { howr }\end{array}$ & \\
\hline $\begin{array}{l}\text { J. T. } \\
\text { W. } \\
\text { P. D. }\end{array}$ & $\begin{array}{l}35 \\
33 \\
32\end{array}$ & $\begin{array}{l}160 \\
128 \\
118\end{array}$ & $\begin{array}{r}126 \\
75\end{array}$ & $\begin{array}{l}83 \\
28 \\
15\end{array}$ & $\begin{array}{r}48 \\
7\end{array}$ & $\begin{array}{r}27 \\
0 \\
6\end{array}$ & $\left|\begin{array}{r}16 \\
0 \\
0\end{array}\right|$ & $\begin{array}{l}5.27 \\
5.20\end{array}$ & $\begin{array}{l}2.2 \\
5.02 \\
6.85\end{array}$ & $\begin{array}{l}30 \\
16\end{array}$ & $\begin{array}{r}57 \\
0\end{array}$ & $\begin{array}{l}3+ \\
0 \\
+\end{array}$ & $\begin{array}{c}2.50 \\
17.5 \\
1.1\end{array}$ & $\begin{array}{l}3.7 \\
4.7 \\
5.5\end{array}$ & $\begin{array}{l}2.5 \\
2.5 \\
1.9\end{array}$ & $\begin{array}{l}0.47 \\
0.52\end{array}$ & $\begin{array}{l}\text { Fatty liver with jaundice } \\
\text { Obstructive jaundice } \\
\text { Neurasthenia, } 3 \text { months }\end{array}$ \\
\hline $\begin{array}{l}\text { T. C. } \\
\text { J. R. }\end{array}$ & $\begin{array}{l}33 \\
25\end{array}$ & $\begin{array}{l}149 \\
158\end{array}$ & $\begin{array}{r}65 \\
113\end{array}$ & $\begin{array}{l}10 \\
71\end{array}$ & $\begin{array}{r}4 \\
50\end{array}$ & $\begin{array}{r}\mathbf{0} \\
\mathbf{3 5}\end{array}$ & $\begin{array}{l}\mathbf{0} \\
\mathbf{0}\end{array}$ & $\begin{array}{l}5.32 \\
2.24\end{array}$ & $\begin{array}{l}7.9 \\
2.65\end{array}$ & $\begin{array}{l}18 \\
15\end{array}$ & $\begin{array}{r}0 \\
10\end{array}$ & $\begin{array}{l}0 \\
+\end{array}$ & $\begin{array}{l}1.85 \\
1.00\end{array}$ & $\begin{array}{l}5.5 \\
1.4\end{array}$ & $\begin{array}{l}1.7 \\
1.6\end{array}$ & 0.74 & $\begin{array}{l}\text { arter acute nepatitis } \\
\text { Essential bilirubinemia } \\
\text { Malnutrition; resected } \\
\text { Ca. of ampulla of Vater; }\end{array}$ \\
\hline $\begin{array}{l}\text { R. W. } \\
\text { M. B. }\end{array}$ & $\begin{array}{l}29 \\
22\end{array}$ & $\begin{array}{l}177 \\
103\end{array}$ & $\begin{array}{r}115 \\
51 *\end{array}$ & $\begin{array}{l}57 \\
21\end{array}$ & $\begin{array}{r}15 \\
7\end{array}$ & $\begin{array}{l}4 \\
1\end{array}$ & $\begin{array}{l}2 \\
0\end{array}$ & $\begin{array}{l}2.22 \\
2.92\end{array}$ & $\begin{array}{l}3.4 \\
5.3\end{array}$ & $\begin{array}{l}17 \\
17\end{array}$ & $\begin{array}{l}\mathbf{0} \\
\mathbf{3}\end{array}$ & $0^{+}$ & $\begin{array}{l}0.85 \\
0.40\end{array}$ & $\begin{array}{l}4.9 \\
2.6\end{array}$ & $\begin{array}{l}2.5 \\
3.4\end{array}$ & & $\begin{array}{l}\text { Diabetes mellitus } \\
\text { Hy poproteinemia in } \\
\text { chronic exfoliative der- }\end{array}$ \\
\hline $\begin{array}{l}\text { E. A. } \\
\text { J. H. }\end{array}$ & $\begin{array}{l}30 \\
\mathbf{3 3}\end{array}$ & $\begin{array}{l}189 \\
185\end{array}$ & $\begin{array}{r}76 \\
127\end{array}$ & $\begin{array}{l}21 \\
73\end{array}$ & $\begin{array}{l}13 \\
45\end{array}$ & $\begin{array}{c}7 \\
22.5\end{array}$ & $\mathbf{0}$ & $\begin{array}{l}5.28 \\
2.46\end{array}$ & $\begin{array}{l}7.3 \\
3.07\end{array}$ & $\begin{array}{l}15 \\
17\end{array}$ & $\begin{array}{c}23 \\
2.5\end{array}$ & $\stackrel{+}{0}$ & $\begin{array}{l}1.2 \\
0.50\end{array}$ & $\begin{array}{l}5.0 \\
3.4\end{array}$ & $\begin{array}{l}2.3 \\
2.8\end{array}$ & & $\begin{array}{l}\text { Polyneuritis, healed } \\
\text { Chronic glomerulo- }\end{array}$ \\
\hline A. $\mathbf{M}$. & 27 & 109 & 51 & 17 & $\mathbf{0}$ & $\mathbf{0}$ & $\mathbf{0}$ & 1.08 & 6.2 & 22 & 5 & $\mathbf{0}$ & 0.4 & 4.4 & 1.6 & & Secondary carcinoma of \\
\hline A. E. & 28 & 108 & 60 & 13 & 7 & 2 & 0 & 2.86 & 7.1 & 20 & $\mathbf{0}$ & $\mathbf{0}$ & 0.20 & 1.7 & 2.2 & & Amyloid disease of liver \\
\hline
\end{tabular}

* 33 minutes.

(Table I) suffering from rheumatoid arthritis had a 3 + cephalin flocculation reaction which was associated with hyperglobulinemia. Since there was no other evidence of liver dysfunction this patient was included with the control group. However, in 19 instances the diagnosis was confirmed by puncture biopsies of the liver; and in 7 instances by autopsies.

Values for blood galactose and for the G.R.C. are shown on Table I. It may be seen that the value for blood galactose 15 minutes after injection varied from 76 to $200 \mathrm{mgm}$. per $100 \mathrm{ml}$. in different patients. As stated previously, this variation may be due to differences in blood volume and in tissue space volume, as well as to differences in the amount of galactose injected, excreted, and utilized by the body. Values for blood galactose at subsequent 15 -minute intervals also varied widely from patient to patient. In 8 patients the test was repeated twice; in 5 patients, 3 times. Tests in the same patient gave similar curves. Our findings confirm the observation of Bassett, Althausen and Coltrin that normally this quantity of galactose is cleared from the blood stream within 75 minutes of the time of injection. Indeed, most normal subjects show no appreciable galactose in the blood after 45 or 60 minutes.

Differentiation between normals and patients with liver damage is easily made by comparing the curves showing the rate of disappearance of galactose from the blood stream. Whereas the slope of the curve in normals is steep, the curves 
obtained from patients with liver damage are more gradual, indicating a slower disappearance of galactose from the blood stream (Figure 2). Table I shows that the value for the G.R.C. varies

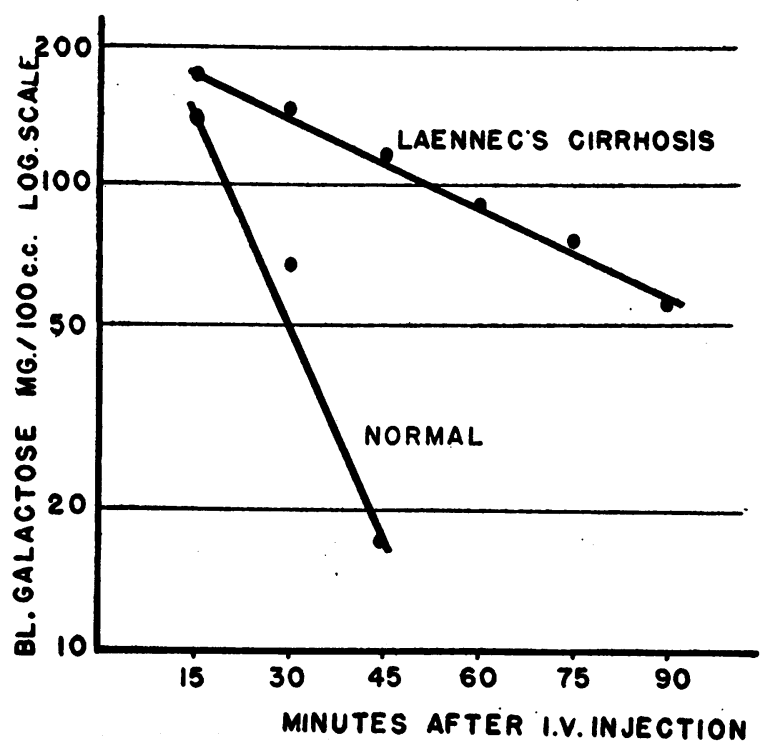

Fig. 2. Comparison of Galactose Removal from the Blood in a Normal adult (H. C.) and a Patient with Decompensated Laennec's Cirrhosis (P. B.) after I.v. InJection of 0.5 Gram PER KGM. from 4.2 to 9.5 in the controls, and is below 4 in patients with parenchymal liver damage.

Figure 3 shows the distribution of values for the G.R.C. in the several groups of patients studied. In 6 normal subjects, and in 4 hospital controls without apparent liver disease, the value was above 4. In 19 patients with decompensated Laennec's cirrhosis, and in 7 cases of "compensated" Laennec's cirrhosis, the G.R.C. was below 4. A similar low value was seen in 10 cases of chronic passive congestion of the liver due to heart failure. In 7 cases of acute hepatitis the G.R.C. was also found to be under 4 .

The last column includes a miscellaneous group of patients with various diseases presumably involving the liver, some with and others without functional impairment.

There are 4 patients who showed subnormal values of the G.R.C.:

Patient $T$, a chronic alcoholic, who was admitted because of jaundice, showed a fatty liver by puncture biopsy, and abnormal values for other liver function tests as shown in Table I. Three years prior to this admission he presented massive ascites requiring many paracenteses.

Patient $\mathrm{R}$ was admitted because of severe malnutrition following resection of carcinoma of ampulla of Vater, and

GALACTOSE REMOVAL CONSTANT IN VARIOUS CONDITIONS

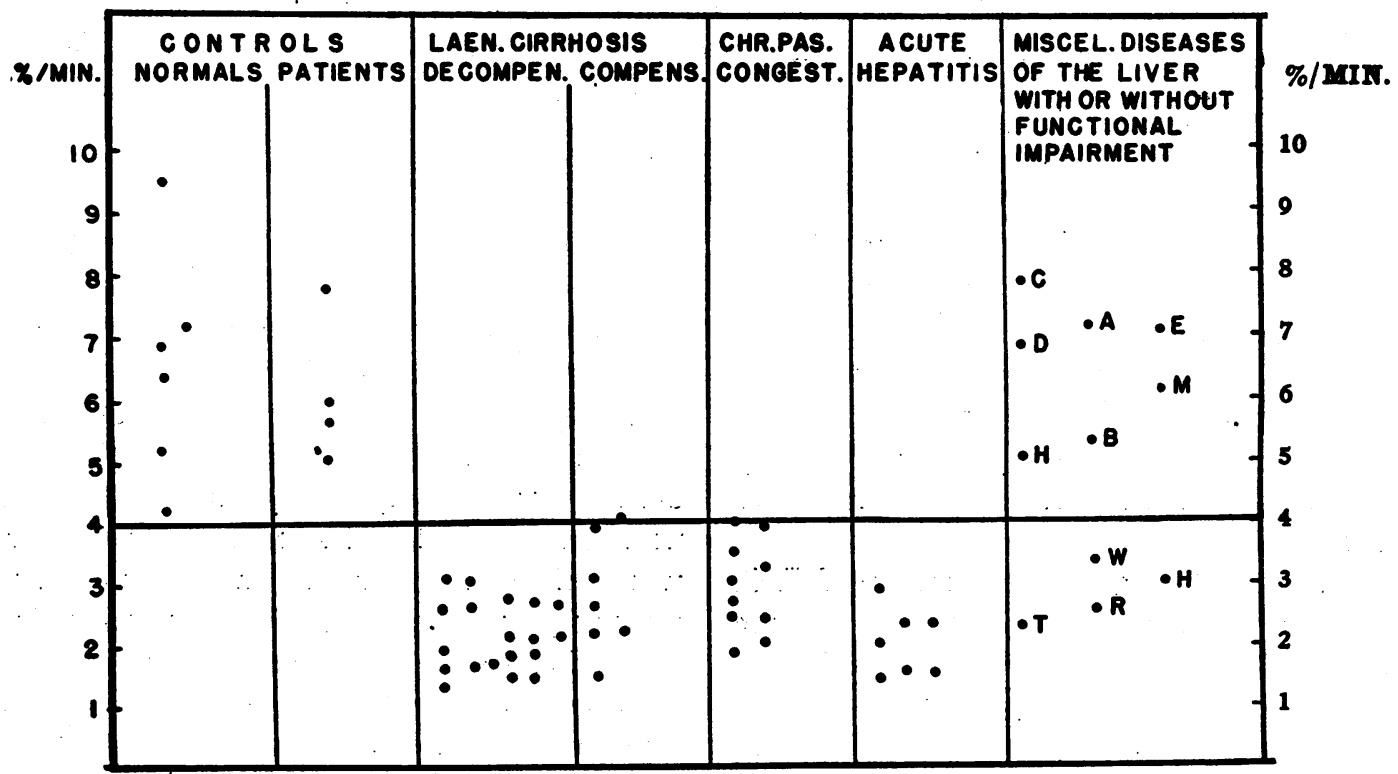

Fig. 3. The Dots Represent the Values of Galactose Removal Constant FOUNd FOR Différent Individuals

The letters in the last column represent the initials of the patient's last name. (See Table. I.) 


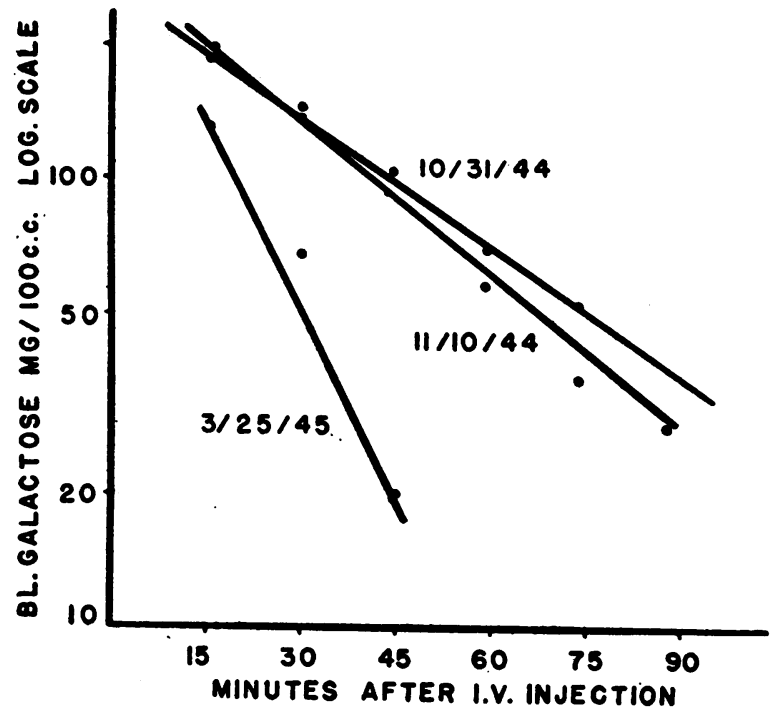

Fig. 4. Galactose Removal from the Blood in a Case of Acute Hepatitis with Recovery

(Patient E. S.) :

$(10 / 31 / 44)$ Second day of jaundice (Serum bilirubin $20 \mathrm{mgm}$. per $100 \mathrm{ml}$.) $\quad$ G.R.C. $=1.90$

(11/10/44) Jaundice had subsided (Serum bilirubin $3 \mathrm{mgm}$. per $100 \mathrm{ml}$.) $\quad$ G.R.C. $=1.94$

(3/25/45) Three months after clinical recovery G.R.C. $=6.2$

because of repeated hemorrhages due to diverticulosis of the duodenum and the large bowel. Autopsy revealed a fatty liver with slight periportal fibrosis.

Patient $H$, suffering from chronic glomerulonephritis, has a history of alcoholism. Hepatomegaly and laboratory signs of liver damage were found 7 years ago. These signs are no longer present.

Patient W, who is a heavy imbiber of alcohol, entered because of a mild diabetes mellitus. He shows no abnormal liver function tests other than the galactose test.

The G.R.C. was normal (above 4 ) in 5 patients who presented diseases of the liver that are usually not associated with functional impairment:

Patient C had "Essential Bilirubinemia," diagnosed 12 years ago. Repeated studies revealed no evidence of liver damage.

Patient D had a mild form of acute hepatitis 3 months prior to the present study. All tests were within normal limits.

Patient $\mathrm{H}$ had obstructive jaundice of 6 weeks' duration.

Patient $M$ had marked hepatomegaly due to metastatic carcinoma.

Patient $\mathrm{E}$ had amyloid disease of liver, kidneys, and spleen.

Two additional cases with normal values of the G.R.C. were included in this group:

Patient A, a chronic alcoholic with diabetes mellitus, was treated 5 years ago for polyneuritis. At that time there was also laboratory evidence of liver damage. At present the only abnormal liver function is bromsulfalein dye retention of 23 per cent at 30 minutes. Liver biopsy shows a normal liver.

Patient $B$, suffering from chronic exfoliative dermatitis with hypoalbuminemia, shows no evidence of functional impairment of the liver.

Urinary excretion of galactose in different individuals varied between 1.08 and 6 grams during the 4-hour period following the rapid intravenous injection. The greater part was excreted during the first hour. It has not been feasible to study in detail the rate of excretion since this would have necessitated an indwelling catheter. There was no correlation between the urinary excretion of galactose and the clinical status of the patients.

\section{Comparison of Galactose Removal Constant with results of other liver function tests}

A series of determinations pertaining to liver functions were performed simultaneously with the galactose test on all subjects. These included serum protein partitions, serum bilirubin, plasma prothrombin, cephalin-cholesterol flocculation, bromsulfalein retention, and urinary urobilinogen excretion.

The bromsulfalein dye test was done according to the procedure of Rosenthal and White (13) employing $5 \mathrm{mgm}$. dye per kgm., and taking the end point at 30 minutes. Determination of the concentration of the dye in the serum was made in the Klett colorimeter with filter no. 54 . Retention of over 10 per cent of dye was considered to be abnormal. Serum proteins were determined by the microKjeldahl method; serum bilirubin, by the method of Evelyn and Malloy (14); cephalin-cholesterol, by the method of Hanger (15) ; and prothrombin, after the method of Quick (16), using "Stypven" (Burroughs-Wellcome) as the thromboplastic agent. Normal values for prothrombin time ranged from 14 to 18 seconds by this method. Urobilinogen was determined by the simplified method of Watson (17). Excretion of more than $0.5 \mathrm{mgm}$. per hour was considered to be abnormal.

The results are shown in columns 11 to 18 , Table I. There was a good correlation between the values of G.R.C. and other liver function tests. In all instances where parenchymal liver damage was indicated by the other tests, the values of the G.R.C. were below 4 .

The galactose test seems to be as sensitive as the bromsulfalein dye retention test for the detection of liver damage, and it has the added advan- 
take of being feasible in the presence of jaundice. Technically, of course, it is more difficult. In the absence of jaundice these 2 tests can be performed simultaneously.

Both the bromsulfalein test and the G.R.C. show persistent abnormality in Laennec's cirrhosis, even though the patient may show signs of considerable clinical improvement. In other words, there may be loss of ascites and jaundice, gain in strength and return to normal activity without comparable improvement in these tests. The significance of this finding is not clear. It is possible that these functional changes result from structural changes in the vascular bed of the liver.

\section{DISCUSSION}

The Galactose Removal Constant gives an overall measure of the disappearance of this substance from the blood stream. This disappearance involves several mechanisms, namely, diffusion into the extracellular fluid compartment of the body, utilization of galactose, and excretion in the urine. In a detailed experiment on 5 dogs, Dominguez and Pomerene (18) analyzed these separate aspects of galactose metabolism. After the rapid intravenous injection of various amounts of galactose, they determined the values for this substance in the blood and urine at frequent intervals over a period of 6 to 7 hours. Their data show that the rate of excretion is proportional to the plasma concentration, and is independent of the amount injected . Constants were also derived for the rate of utilization. Whereas their work provides precise information, the procedure and the formulae are somewhat too elaborate for clinical application.

In the present study it is assumed that a state of equilibrium has been reached between galactose in the blood stream and the galactose in the tissue spaces within the first 15 minutes of injection, and that the disappearance curve thereafter gives an index of galactose utilization. The rate of excretion is not taken into account, since under the present conditions only about 10 per cent of the injected galactose is recovered from the urine. Moreover, since there appears to be little difference between normals and patients with liver damage in the urinary excretion of galactose over a 4-hour period, the neglect of this factor would seem to be of little practical consquence.

\section{SUMMARY AND CONCLUSIONS}

1. The rate of disappearance of galactose from the blood stream after intravenous injection of $0.5 \mathrm{mgm}$. per $\mathrm{kgm}$. was studied in normal adults, patients with Laennec's cirrhosis of the liver, chronic passive congestion of the liver, hepatitis, and other affections of the liver with and without functional impairment.

2. It is suggested that the per cent of galactose removed per minute be termed the Galactose Removal Constant.

3. A simplified formula is given by which the Galactose Removal Constant can be calculated from 2 blood specimens taken 15 and 45 minutes after intravenous injection of galactose.

4. The Galactose Removal Constant varied between 4.2 and 9.5 in the control group, whereas it was below 4 in patients with impaired liver functions.

5. There was a good correlation between the changes in the Galactose Removal Constant and tests pertaining to other liver functions.

The authors wish to acknowledge with thanks the technical assistance of Mrs. Anne Colcher and Mrs. Greta Heinemann.

\section{BIBLIOGRAPHY}

1. Bauer, R., Weitere Untersuchungen über Alimentare Galactosurie. Wien. med. Wchnschr., 1906, 56, 25.

2. Mann, F. C., and Magath, T. B., Studies on the physiology of the liver. Arch. Int. Med., 1922, 30, 73 and 171 .

3. Foster, G. L., Studies on carbohydrate metabolism. J. Biol. Chem., 1923, 55, 291 and 303.

4. Bollman, J. L., Mann, F. C., and Power, M. H., Utilization of galactose following complete removal of liver. Am. J. Physiol., 1935, 111, 483.

5. Shay, H., Schloss, E. M., and Bell, M. A., Metabolism of galactose. Arch. Int. Med., 1931, 47, 391.

6. Roe, J. H., and Schwartzman, A. S., Galactose tolerance as a measure of liver function. Am. J. M. Sc: 1933, 186, 425.

7. Deuel, Harry J., Jr., Intermediary metabolism of fructose and galactose. Physiol. Rev., 1936, 16, 173.

8. Jankelson, I. R., Segal, M., and Aisner, M., Intravenous galactose liver function test. Am. J. Digest. Dis. and Nutrition, 1937, 3, 889.

9. King, E. J., and Aitken, R. S., An intravenous galactose-tolerance test. Lancet, 1940, 2, 543. 
10. Bassett, A., Althausen, T., and Coltrin, G., A new galactose test for differentiation of obstructive from parenchymatous jaundice. Am. J. Digest. Dis., 1941, 8, 432.

11. Hawk, P. B., and Bergeim, O., 11th Edition, 1937, p. 435. P. Blakiston's Son and Co., Inc., Philadelphia.

12. Raymond, A. L., and Blanco, J. G., Blood sugar determination and separation of sugars with live yeast. J. Biol. Chem., 1928, 79, 649.

13. Rosenthal, S. M., and White, E. C., Clinical application of the bromsulphalein test for hepatic function. J. A. M. A., 1925, 84, 1112.

14. Malloy, H. T., and Evelyn, K. A., The determination of bilirubin in the photoelectric colorimeter J. Biol. Chem., 1937, 119, 481.
15. Hanger, F. M., Flocculation of cephalin-cholesterol emulsions by pathological sera. Tr. A. Am. Physicians, 1938, 53, 148.

16. Quick, A. J., The clinical application of the hippuric acid and the prothrombin tests. Am. J. Clin. Path., 1940, 10, 222.

17. Watson, C. J., Schwartz, S., Sborov, V., and Bertie, E., Studies of urobilinogen: A simple method for the quantitative recording of the Ehrlich reaction as carried out with urine and feces Am. J. Clin. Path., 1944, 14, 605.

18. Dominguez, R., and Pomerene, E., Kinetics of the disappearance of galactose from plasma after rapid intravenous injection. Am. J. Physiol., 1944, 141, 368. 